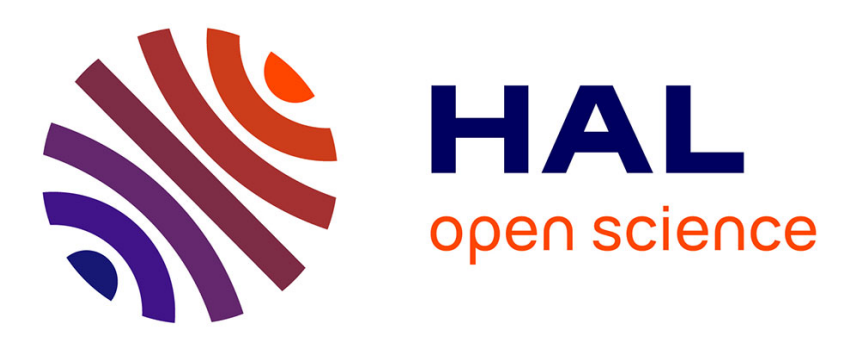

\title{
Multimodal reduction of acoustic radiation of thin plates by using a single piezoelectric patch with a negative capacitance shunt
}

Charlie Bricault, C. Pezerat, M. Collet, A. Pyskir, P. Perrard, G. Matten, Vicente Romero-García

\section{To cite this version:}

Charlie Bricault, C. Pezerat, M. Collet, A. Pyskir, P. Perrard, et al.. Multimodal reduction of acoustic radiation of thin plates by using a single piezoelectric patch with a negative capacitance shunt. Applied Acoustics, 2019, 145, pp.320-327. 10.1016/j.apacoust.2018.10.016 . hal-02109901

\section{HAL Id: hal-02109901 https://hal.science/hal-02109901}

Submitted on 16 Sep 2019

HAL is a multi-disciplinary open access archive for the deposit and dissemination of scientific research documents, whether they are published or not. The documents may come from teaching and research institutions in France or abroad, or from public or private research centers.
L'archive ouverte pluridisciplinaire HAL, est destinée au dépôt et à la diffusion de documents scientifiques de niveau recherche, publiés ou non, émanant des établissements d'enseignement et de recherche français ou étrangers, des laboratoires publics ou privés. 


\title{
Multimodal reduction of acoustic radiation of thin plates by using a single piezoelectric patch with a negative capacitance shunt
}

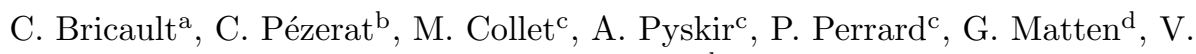 \\ Romero-García ${ }^{b}$ \\ ${ }^{a}$ Institut de Recherche Technologique Jules Verne, LAUM UMR 6613, Av. O. Messiaen, \\ 72085 Le Mans, France \\ ${ }^{b}$ Laboratoire d'acoustique de l'Université du Mans, Le Mans Université, CNRS, LAUM \\ UMR 6613, Av. O. Messiaen, 72085 Le Mans, France \\ ${ }^{c}$ École Centrale de Lyon, CNRS, LTDS UMR 5513, Av. G. De Collongue, 69134 Ecully, \\ France \\ ${ }^{d}$ FEMTO-ST Institute, Department of Applied Mechanics, Besançon, France
}

\begin{abstract}
The ability of a single piezoelectric patch with a single negative capacitance shunt for the multimodal vibration damping is used in this work to drastically reduce the acoustic radiation of a vibrating plate. With a geometry fixed, the elastic properties can be controlled using the resistance and the negative capacitance of the shunt: using a value of the negative capacitance close to the instability domain of the circuit, the stiffness can be placed in the softening region of the system, while the resistance tunes both the stiffness and losses. By means of an optimal design, several modes with non-zero electromechanical coupling factors can be simultaneously damped. As a consequence at these frequencies the acoustic radiation of the plate is drastically decreased. The results open prospects to tackle the vibration and acoustic problems in an industrial context.
\end{abstract}

Keywords: Acoustic radiation suppression, Vibration damping, Negative capacitance

Preprint submitted to Applied Acoustics

September 16, 2019 


\section{Introduction}

The design of lightweight materials with robust mechanical properties and reduced acoustic radiation is a scientific and technological challenge in several fields as in automotive, aerospace or naval industries. While lightweight struc5 tures could considerably reduce the environmental impacts, their reduction of mass inevitably leads to acoustic and vibration issues. Therefore, vibrations damping and in particular the suppression of the sound radiation from structures with lightweight absorbers is a relevant topic of increasing interest.

Different possibilities have been proposed to damp vibrations. For instance, tuned mass dampers [1, locally resonant phononic crystals [2, pillar-based phononic crystals 3, 4, or phononic crystals composed by piezoelectric or piezomagnetic materials [5, 6, 7]. However, systems using piezoelectric shunted damping circuits $[8,9,10,11,12,13,14,15,16,17$, introduce small additional mass with a high tunability by only adjusting the properties of the shunt. The pioneering work of Hagood and von Flotow [9] showed how a single shunted piezoelectric patch acts as a vibration absorber at a single frequency. The most used shunts are the resistive (single resistance) and the resonant (resistance and inductance in series) ones [10. Numerous works have been published [18, 19, 20, 21] that present analysis of the capability and efficiency of a single shunted piezoelectrical patch for structural stabilization and wave cancellation at a single frequency. Extensions have been developed to design more complex shunting circuits to extend the effectiveness over broader frequency bands, using for example the multimodal shunts [11, 12, 22, 23, 24]. Periodic distributions of such piezoelectric shunted patches have also been proposed. Applications to mitigate vibrations in a beam [25, 26] or a plate [13] over broad frequency bands as well as to control the wave propagation in a thin plate [14 have been studied. Other methods of broadband suppression include state switching [27, 28, 29] or synthetic impedance [30] among others.

Recently, shunt circuits with negative capacitance are receiving increasing interest due to their ability for improving the vibration control [31, 32, 33, 34, 
35. 36]. Specially, attenuation performance of the shunt can be significantly increased [32, 37. This kind of systems increases the value of the effective electromechanical coupling factor (EEMCF), resulting in better attenuations [33. However, the active nature of such electromechanical systems leads to regions of instabilities described by De Marneffe and Preumont [32]. It is worth noting here that the closest the circuit is to the stability limit, the highest vibration attenuation performance appears if the structure is in the softener domain 33, 37. It is worth noting here that the "softener" and the "stiffening" domains are expressions which can be used in the vibration damping terminology with piezoelectric patches shunted with a negative capacitance circuit [38, 39. The first domain denotes the region in which the stiffness decreases producing a shift to the low frequencies of the eigenfrequencies. The second domain corresponds to the opposite phenomenon.

When sound radiation is considered, the control of the vibration components which radiate sound most efficiently is crucial for the reduction of the radiated sound. In this sense, noise control techniques are often focussed on reducing the amplitudes of vibration by either including damping treatments or using structural modification to mitigate the sound radiation. Passive solutions to tackle the radiation problem have been used [40, 41, 42, however, these approaches 50 tend to be efficient only for high frequencies and they lead to a significant mass increase. There is also a large interest for noise reduction systems based on piezoelectric shunts 43, 44, 45, 46, 47, 48, 49. These systems are likely to compensate the lack of efficiency of passive methods and usually add less mass to the overall system.

In this work, we use a single piezoelectric patch with a negative capacitance shunt, working close to the instability domain in order to show the possibility to damp simultaneously different modes of an aluminum square plate and reduce its acoustic radiation. To do that we use the concept of the multi-mode damping by means of negative capacitance controller (already introduced by ${ }_{60}[33,37$ ) to propose a general procedure to obtain the optimal values of $R$ and $C_{n e g}$ that minimizes the vibrations of the system and the acoustic radiation in 
(a)

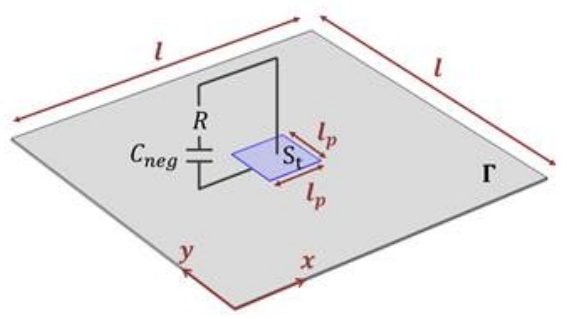

(b)

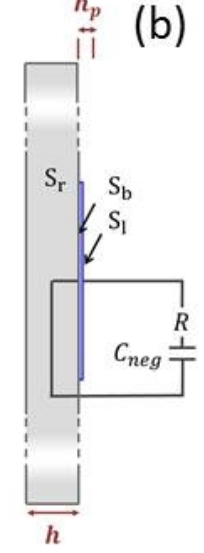

Figure 1: (Color online) (a) Top and (b) lateral schemas of the studied system: an aluminum plate with a piezoelectric patch PZ26 with a negative capacitance shunt. Values of the physical parameters are shown in the Appendix A

terms of given objectives. In difference with the previous works we go further and use this configuration to prove that the acoustic radiation is drastically reduced by using these optimal values due to the fact that the kinetic energy of the modes is reduced and therefore the power acoustic radiation is drastically attenuated. The objective of this work is twofold: to investigate the effect of the negative capacitance shunt on both the vibration a rectangular plate and its sound radiation. Once the geometry is fixed, we choose a frequency range in which several modes with non-zero EEMCF are excited. Then, the elastic properties of the whole system can be controlled by tuning the resistance and the negative capacitance of the shunt: by changing the negative capacitance, the stiffness can be placed in the softener domain, close to the instability of the shunt where better performances are predicted; the resistance is used to tune the additional losses to reduce the vibration amplitude of the modes. Therefore the sound radiation by the plate can be drastically reduced for these modes. This work shows a procedure to obtain the values of the electrical components of the shunt to simultaneously damp several modes of the system with a single shunted patch, showing a strong reduction of the sound radiation. The problem 
is numerically solved and experimentally tested with good agreement.

\section{Setup and governing equations}

A schematic representation of the studied system is shown in Fig. 1 It consists of an isotropic aluminum plate with $l=300 \mathrm{~mm}$ and $h=2 \mathrm{~mm}$ on which a piezoelectric patch is placed in the center. The dimensions of the ceramic piezoelectric patch PZ26 are $l_{p}=50 \mathrm{~mm}$ and $h_{p}=0.5 \mathrm{~mm}$. The electrodes of the patch are linked by a circuit in series with an impedance $Z_{s h}=R+1 /\left(j \omega C_{n e g}\right)$, where $R$ represents the resistance and $C_{n e g}$ is the capacitance of the shunt. We chose the series circuit because the damping produced by this kind of circuit is bigger than the parallel one [33].

The dynamic behavior of this system is driven by the following partial derivative equations

$$
\begin{array}{cl}
\rho \ddot{\boldsymbol{w}}(\boldsymbol{r}, t)-\nabla \sigma(\boldsymbol{r}, t)=0, & \forall \boldsymbol{r} \in \boldsymbol{\Gamma}, \\
-\boldsymbol{\nabla} \boldsymbol{D}(\boldsymbol{r}, t)=0, & \forall \boldsymbol{r} \in \boldsymbol{\Gamma},
\end{array}
$$

where $\rho$ is the spatially dependent density, $\boldsymbol{w}(\boldsymbol{r}, t)$ is the displacement vector, $\sigma$ represents the spatially dependent Cauchy stress tensor and $\boldsymbol{D}$ the spatially dependent electrical displacement. $\boldsymbol{\Gamma}$ refers to the global system domain. The piezoelectric material behavior is given by

$$
\begin{gathered}
\boldsymbol{\sigma}=C^{E}(\boldsymbol{r}) \boldsymbol{S}-e^{t}(\boldsymbol{r}) \boldsymbol{E} \\
\boldsymbol{D}=e(\boldsymbol{r}) \boldsymbol{S}+\varepsilon^{S}(\boldsymbol{r}) \boldsymbol{E}
\end{gathered}
$$

where $\boldsymbol{E}=-\nabla V$ is the electric field vector, $V$ being the voltage, $C^{E}$ the elasticity tensor at constant electric field, $e^{T}$ the piezoelectric coupling coefficients tensor, $\boldsymbol{S}=1 / 2\left[\nabla \boldsymbol{w}^{t}(\boldsymbol{r}, t)+\boldsymbol{w}(\boldsymbol{r}, t) \nabla^{t}\right]$ the Green strain tensor $(t$ in this expression is the mathematical transpose operation) and $\varepsilon^{S}$ the dielectric permittivity at constant strain. We take into account the electrical shunt, considering harmonic excitation of the type $e^{j \omega t}$, with another expression for the electric charge 
generated by the piezoelectric patch in the electrical circuit

$$
q(\omega)=\int i d t=i /(j \omega)=-\int_{S_{t}} \boldsymbol{D} \cdot \boldsymbol{n} d S,
$$

where $i$ is the current, $\boldsymbol{n}$ means the normal vector to the top electrode surface $S_{t}$. A potential $V(\omega)=Z_{s h}(\omega) j \omega q(\omega)$ is applied on the top electrode depending on the total charge $q(\omega)$ defined in Eq. (5).

The mechanical boundary conditions are considered free and the electrical boundary conditions included in the formulation are

$$
\begin{array}{cc}
V(\boldsymbol{r}, \omega)=0, & \forall \boldsymbol{r} \in S_{b}, \\
V(\boldsymbol{r}, \omega)=V(\omega), & \forall \boldsymbol{r} \in S_{t}, \\
\operatorname{D.n}(\boldsymbol{r}, \omega)=0, & \forall \boldsymbol{r} \in S_{l},
\end{array}
$$

where $S_{b}$ is the grounded bottom area of the piezoelectric patch and $S_{l}$ is the lateral electrode area. The theoretical analysis of the system is done by solving these coupled equations with a Finite Element Method using COMSOL Multiphysics ${ }^{\circledR}$ Software. We solve both the eigenvalue problem of the system in order to have the modal response of the whole system as well as the forced problem by introducing a point force source in the domain.

The acoustic radiation of the plate with the piezoelectric shunt can be calculated theoretically from the total energy radiated to the far-field [50]. We discretize the plate with $I$ elements, therefore by using the Rayleigh method we can calculate the radiated pressure over a semi-sphere of radius $r$ discretized with $N$ points. The pressure radiated by the plate to the $n$-th element of the semi-sphere is given by

$$
p_{\omega, n}=-\imath k \rho_{0} c_{0} \sum_{i=1}^{I} \frac{e^{\imath k R_{i, n}}}{2 \pi R_{i, n}} v_{\omega, i}(x, y) d x d y,
$$

where $R_{i, n}$ presents the distance of the $i$-th element in the vibration plate to the $n$-th element of the semi-sphere, $v_{\omega, i}$ is the point vibration velocity of the $i$-th element at angular frequency $\omega$ and $k, \rho_{0}$ and $c_{0}$ are the wavevector, the density and the sound velocity in the air respectively. Therefore, the radiated 
power is calculated as

$$
W_{a}=\sum_{n=1}^{N} \frac{\left|p_{\omega, n}\right|^{2}}{\rho_{0} c_{0}} r^{2} \sin \theta d \theta d \phi .
$$

\section{Optimal capacitance and resistance}

We start by making the modal analysis of the complete shunted electromechanical system, which depends on the properties of the shunted circuit, i.e., on $R$ and $C_{n e g}$. We will pay attention to a range of low frequencies where several modes of the system coexist, here from 1300 to $2000 \mathrm{~Hz}$. As shown in Fig. 2(a), this system presents three modes in this region (mode A: $1550 \mathrm{~Hz}$, mode B:

$1710 \mathrm{~Hz}$ and mode C: $1960 \mathrm{~Hz}$ ) with non-zero EEMCF defined by [10, 33 .

$$
k_{e f f, i}=\sqrt{\frac{\omega_{o c, i}^{2}-\omega_{s c, i}^{2}}{\omega_{s c, i}^{2}}},
$$

where $\omega_{o c, i}$ is the pulsation of the mode $i$ with the shunt in open-circuit case and $\omega_{s c, i}$ in short circuit case. Open-circuit condition is equivalent to applying an infinite impedance $Z_{s h}=\infty$, while the short circuit condition is equivalent to applying a zero impedance $Z_{s h}=0$. To make an open circuit condition with the numerical model, it is applied a $V=0 \mathrm{~V}$ condition only the grounded electrode $\left(\forall \boldsymbol{r} \in S_{b}\right)$, and to make a short circuit condition it is applied a $V=0$ $\mathrm{V}$ condition on the both electrodes $\left(\forall \boldsymbol{r} \in S_{b}\right.$ and $\left.\forall \boldsymbol{r} \in S_{t}\right)$. This indicator represents the level of controllability through the piezoelectric coupling. The greater the coefficient the stronger the piezoelectric authority.

When this parameter is non-zero for a mode with the natural pulsation $\omega_{i}$, the displacement of this mode can be controlled [10, 33]. For these three modes an optimal couple $\left(R, C_{n e g}\right)$ will be determined to simultaneously attenuate their modal amplitudes.

Before starting our optimization procedure, we should analyze the instability condition of the system in order to determine what are the limits of the physical values for the $C_{n e g}$. We have revisited the instability condition following the reference 32,33$]$. There are two important capacitances to analyze in order to 
know the instability condition of the system. The first one is the capacitance of the isolated patch, $C_{p}^{T}=S \varepsilon_{33}^{T} / h$ (where $\varepsilon_{33}^{T}=\varepsilon_{33, r}^{T} \varepsilon_{0}$, with $\varepsilon_{0}=8.85410^{-12}$ ), defined as the zero-frequency limit of the $C_{n e g}$ of the isolated patch; the second one is the capacitance of the coupled system patch-plate, $C_{0}$, defined as the zero frequency limit of the $C_{n e g}$ of the coupled system. Figure 3 shows the dependence of the negative capacitance on the frequency. For the isolated system, the $C_{p}^{T}$ shows the instability limit of the isolated patch, while $C_{0}$ shows the instability condition of the coupled system. Then the instability condition of the coupled system is given by $\left|C_{n e g}\right|>C_{0}$. In our system $C_{p}^{T}=48.6 \mathrm{nF}$ and $C_{0}=42.7 \mathrm{nF}$ are the obtained numerical values and therefore the instability condition reads as $\left|C_{n e g}\right|>42.7 \mathrm{nF}$.

In a first step, we analyze the vibrational problem. By considering $R=0$, we study the dependence of the real part of the complex frequencies of the modes (they are complex due to the structural damping, but with a very small imaginary part) on the values of the negative capacitance (see Fig. 2(b)). The range of variation of $C_{n e g}$ is chosen in the softener domain of the coupled system and we chose $C_{n e g}=-4310^{-9} \mathrm{~F}$ which is close to the instability region. The instability $\left|C_{n e g}\right|>C_{0}$ condition is respected. The second step consists in computing the real and imaginary parts of the frequencies of the modes with $C_{n e g}=-4310^{-9} \mathrm{~F}$ for different values of the resistance $R$ (in this case from 1 to $250 \Omega$ ). As soon as the resistance is introduced in the system, the eigenvalues are complex due to the presence of damping in the system. Figure 2(c), shows the evolution of the damping parameter, $\xi=\Im\left(\omega_{i}\right) / \Re\left(\omega_{i}\right)$, for each mode as the resistance increases (arrow direction in the different lines). For each mode, a maximum value of the damping parameter, $\xi_{\max }$, can be reached. Figure 2(d), following the left vertical axis, shows the dependence of the normalized damping, defined as $\Xi_{I}=\xi_{I} / \xi_{\max , I}(I=\mathrm{A}, \mathrm{B}, \mathrm{C})$ on $R$. Each mode presents a maximum 175 damping at different values of $R$ in agreement with Fig. 2(c): $R=28.75 \Omega$ for the mode A, $R=50 \Omega$ for the mode B and $R=43.75 \Omega$ for the mode C. In order to reach a global effective damping of the three modes, a compromise should be established: the value allowing to maximize the damping effect for 

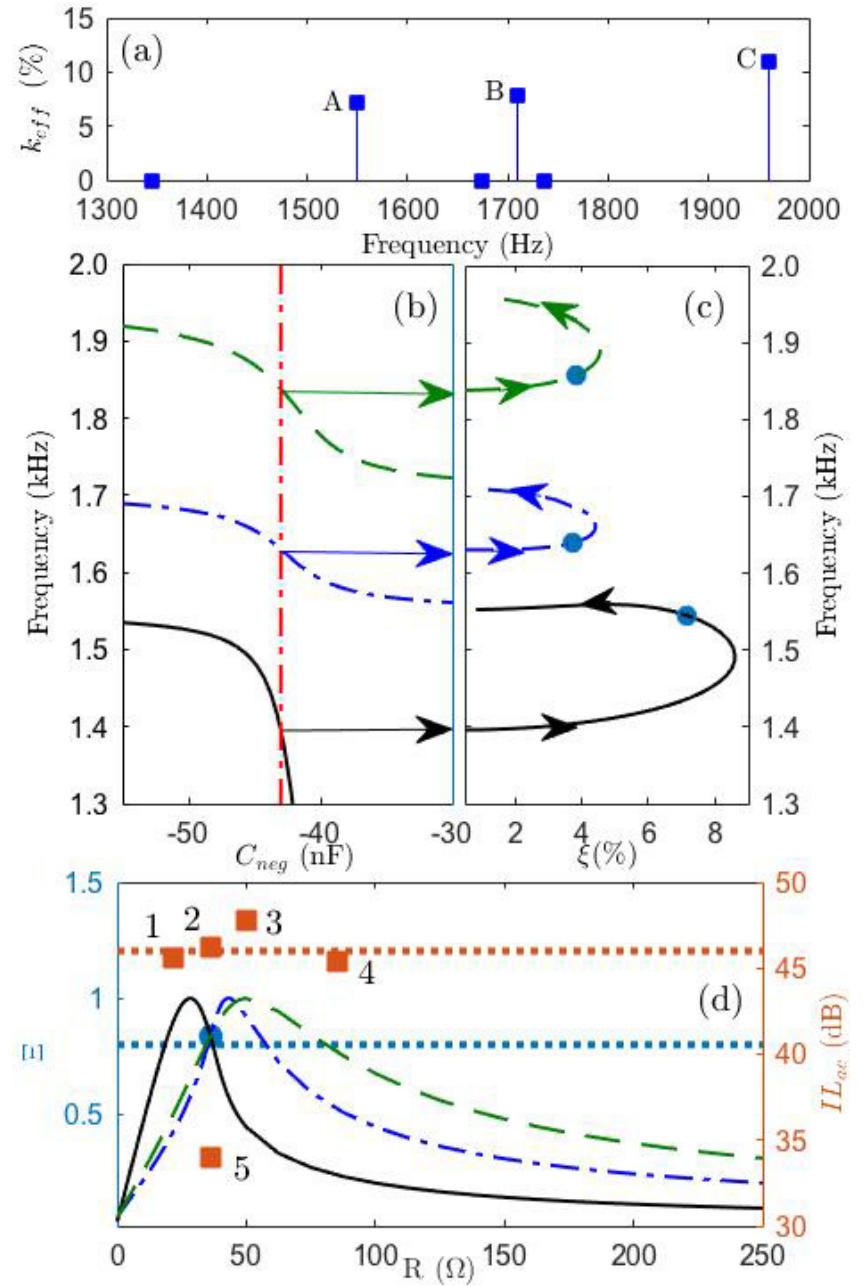

Figure 2: (Color online) Numerical analysis of the modal response of the system. (a) Effective electromechanical coupling factor of the modes. (b) Dependence of the eigenfrequencies of the modes A (black continuous line), B (blue dot-dashed line) and C (green dashed line) on the negative capacitance $C_{n e g}$ (with $R=0$ ). Dot dashed line represents the negative capacitance value chosen in the softener domain and closed to the instability region ; $C_{n e g}=-4310^{-9} \mathrm{~F}$. (c) Dependence of the real eigenfrequencies and the damping factor $\xi$ on the different values of resistance $R=[1,250] \Omega$ (with $C_{n e g}=-4310^{-9} F$ ). Arrows shows the direction of increasing $R$. (d) Dependence of the normalized damping parameter $\Xi_{I}$ (with $C_{n e g}=-4310^{-9} F$ ) on the different values of resistance $R=[1,250] \Omega$ (left vertical axis, in blue). Evaluation of the $I L_{a c}$ for the configurations 1-5 in orange squares (right vertical axis, in orange). Blue dotted line marks the threshold of $80 \%$ of vibration damping. Blue dots denote the value of the normalized parameter for the optimal resistance, $R=36.25 \Omega$. Orange dotted line represents the threshold of the $I L_{a c}>46 \mathrm{~dB}$. 


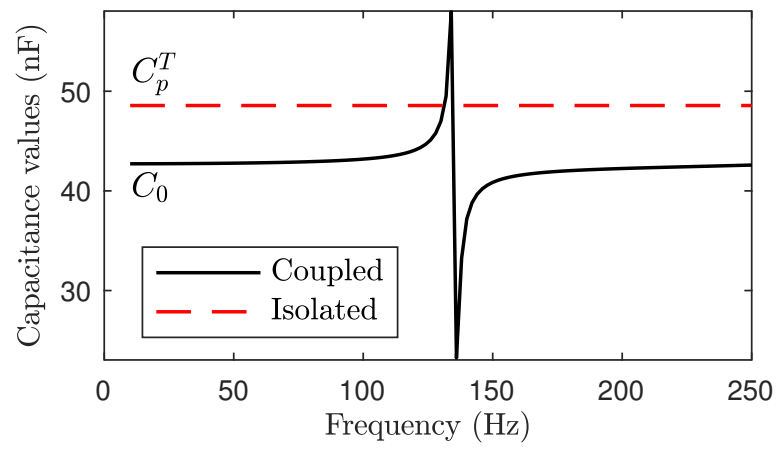

Figure 3: Dependence on the frequency of the negative capacitance of the isolated patch (red dashed line), and of the coupled system (continuous black line) numerically calculated.

the three modes is $R=36.25 \Omega$ (blue dot in Figs. $2(\mathrm{c})-2(\mathrm{~d}))$. The criteria used to chose the optimal for the vibrational problem is stablished as the normalized damping factor should be bigger than $80 \%(\Xi=0.8)$ for the three modes A, B and C simultaneously.

In a second step, we analyze the acoustic radiation problem in order to consider it in the criteria to chose the optimal. From the radiated power, calculated from Eq. 8), we define here the accumulated insertion loss, $I L_{a c}$, defined as,

$$
I L_{a c}=\sum_{i=A, B, C}\left(W_{a}^{S C}-W_{a}^{i}\right)
$$

where $W_{a}^{S C}$ is the radiated power in the short circuit configuration (in dB) and $W_{a}^{i}$ is the radiated power in the full configuration for the mode $i=\mathrm{A}$, B, and C. Figure 2(d) (following the right vertical axis) shows the evaluation of 5 different configurations shown in the Table 1 . The orange dashed line represents the threshold of $46 \mathrm{~dB}$ of accumulated insertion loss. Among the analyzed configurations, there are only two that have accumulated insertion loss bigger than this value, the configurations 2 and 3 .

The optimal values of $R$ and $C_{n e g}$ used to control the modes A, B and $\mathrm{C}$ have been chosen by the configuration that simultaneously fulfill both the optimal 195 criteria for the vibration problem (normalized damping factor bigger than $80 \%$ ) and that of the acoustic problem (accumulated insertion loss bigger than $46 \mathrm{~dB}$ ). 
Table 1: Configurations evaluated for the radiation problem

\begin{tabular}{ccc}
\hline \hline $\begin{array}{c}\text { Configuration } \\
\text { Number }\end{array}$ & $R$ & $\begin{array}{c}C_{\text {neg }} \\
\Omega\end{array}$ \\
\hline \hline 1 & 21.75 & -43 \\
2 & 36.25 & -43 \\
3 & 50 & -43 \\
4 & 85 & -43 \\
5 & 36.25 & -48.6 \\
\hline \hline
\end{tabular}

The optimal values are $R=36.25 \Omega$ and $C_{n e g}=-4310^{-9} \mathrm{~F}$. At this stage it is worth noting that as our numerical model cannot consider the real conditions of the experiment, as it does not take into account the effect of the glue and the possible imperfections of the PZ26, the numerical optimization gives us a good approximation to the optimal values.

\section{Experimental setup}

Figures $4(\mathrm{a})$ and $4(\mathrm{~b})$ respectively show a sketch and a picture of the experimental setup used to validate the previous numerical results. The plate is excited by a shaker emitting a sweep sine in a corner of the plate. The harmonic loading source is measured with an impedance head. The velocity is measured with a Polytech scanning laser vibrometer in the opposite corner of the excitation. The negative capacitance shunt is synthesized by an analog electronic circuit organized around an operational amplifier (OPA) (for more details, see the Appendix B. This circuit allows us to set the negative capacitance close to the instability region by tuning the potentiometers. However, there are several factors that modify the physical parameters of the real system and make difficult to find the same values as in the theory. On one hand, in the experimental set up, the PZ26 is glued to the aluminum plate and this fact is not considered in our simulations. In fact, the effect of the glue on the electromechanical coupling 

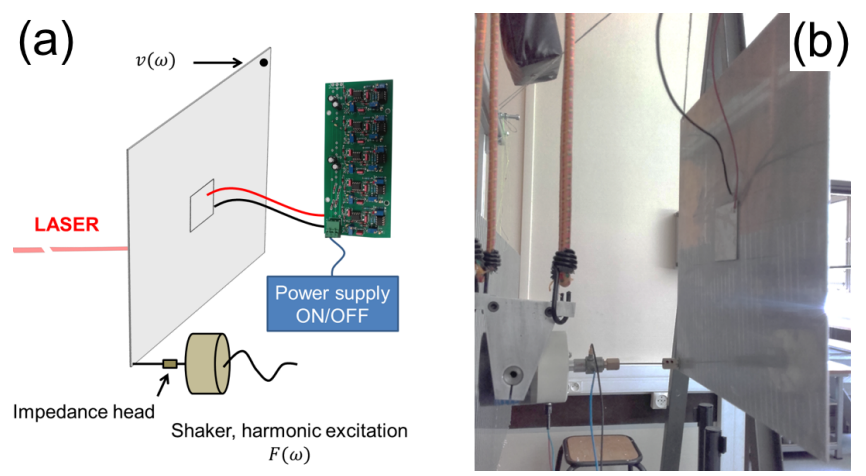

Figure 4: (Color online) (a) Schematic representation and (b) picture of the experimental setup.

of the PZ26 cannot be controlled. On the other hand, the potentiometers have not enough precision to set the negative capacitance and resistance precisely because we are very close to the instability region of the system. The experimental values used in this work are $C_{n e g}^{e x p} \simeq-45.610^{-9} \mathrm{~F}$ and $R^{e x p}=39.9 \Omega$ which are not far from those numerically obtained. They have been found from the values given form the numerical optimization procedure and then look around these values in order to find the good performance in the experimental set-up.

\section{Results}

In order to compare the theoretical results with the experimental measurements we evaluate the mobility, $H(\omega)=\frac{v(\omega)}{F(\omega)}$, that represents the transfer function between the point vibration velocity $v(\omega)$ at some place in the plate with respect to applied force of the harmonic excitation source $F(\omega)$ at the excitation point. Figures 5(a) and 5(b) respectively show the theoretical predictions and the experimental measurements of the mobility in open-circuit case, short circuit case and in case with the optimal resistive and negative capacitance shunt. The presence of the negative capacitance shunt reduces the mobility of the plate around the frequencies of the three selected modes. It is worth noting here that the modes with $k_{\text {eff }}=0$ are not affected by the presence of the shunt. 

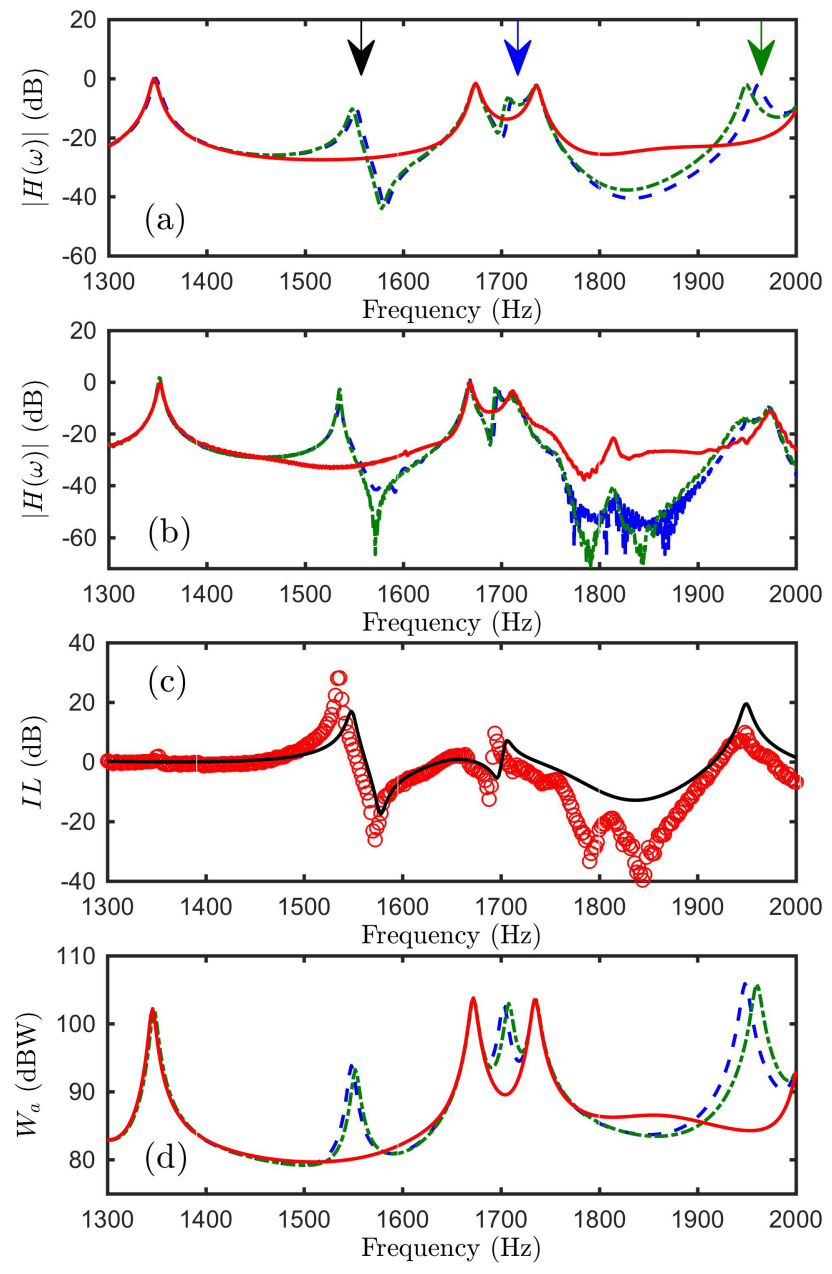

Figure 5: (Color online) (a) Theoretical and (b) experimental mobility functions $H(\omega)$ in open-circuit (blue dashed line), short circuit (green dot-dashed line) and negative capacitance shunt circuit (red continuous line). The parameters in (a) are $C_{n e g}=-4310^{-9} \mathrm{~F}$ and $R=36.25 \Omega$ and in (b) $C_{n e g}^{e x p} \simeq-45.610^{-9} F$ and $R^{e x p}=39.9 \Omega$. (c) Theoretical (black line) and experimental (red circles) IL. (d) Theoretical sound power radiation for the open-circuit (blue dashed line), short circuit (green dot-dashed line) and negative capacitance shunt circuit (red continuous line). 
The attenuation of the system, produced by the presence of the shunted

235 pacitance, is evaluated by using the insertion loss (IL), defined as $20 \log \left(\left|H_{S C}\right| /\left|H_{C_{n e g}}\right|\right)$, where $H_{S C}$ is the mobility in short circuit configuration and $H_{C_{n e g}}$ is the one with the shunt connected to the piezoelectric patch. It represents the reduction of the vibration level brought about by the shunt, compared to the short circuit configuration. Figure 5(c) shows the experimental and the theoretical results. The increase of the attenuation around the eigen frequencies of the plate is evidenced (with more than $3 \mathrm{~dB}$ in average). The discrepancies between the theory and the experiments are due, first to the differences in the values of the parameters of the shunt between the theoretical and experimental circuits 245 (producing mostly a shift in frequencies of the maxima of this ratio); second, to the imperfections in the experimental setup (producing the slightly different magnitudes between the theoretical predictions and the experimental results).

Figure 5(d) shows the radiated power for the three analyzed cases: in opencircuit case, short circuit case and in case with the optimal negative capacitance shunt. The presence of the optimal resistance and the negative capacitance reduces the sound radiation of the plate around the frequencies of the three selected modes. For the three modes we can observe an average reduction of 10 $\mathrm{dB}$ of the acoustic radiation power of each mode. Again, the sound radiation of the modes with $k_{\text {eff }}=0$ is not reduced.

Finally, in order to see the effect in the profiles of vibrations, we have evaluated the theoretical and the experimental maps of mobility defined and measured on a mesh of $29 \times 29$ points, also known as the operational responses of the system, for the mode $\mathrm{A}$ in the controlled and uncontrolled cases (for the other modes, see the Appendix C). Figures 6(a) and 6(c) show respectively the theoretical and experimental maps of mobility for the case without the piezoelectric shunted patch (uncontrolled). The mode distribution is clearly shown in both cases in good agreement. Figures 6(b) and 6(d) respectively represent the theoretical and experimental mobility maps for the controlled case, i.e. with the negative capacitance active. A significant reduction of the mobility is produced. 


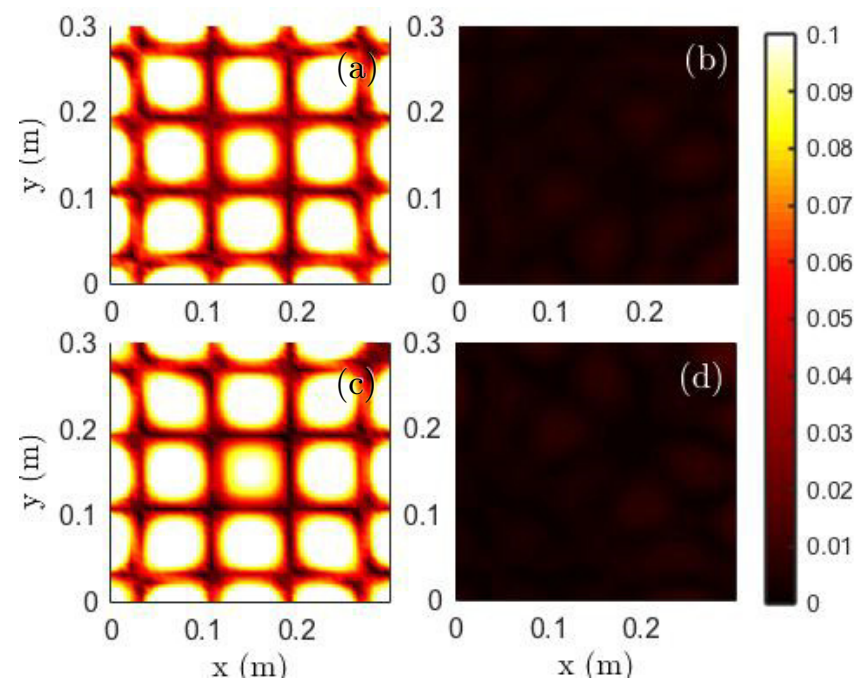

Figure 6: (Color online) Mobility profiles $\left(\mathrm{m} \cdot \mathrm{s}^{-1} / \mathrm{N}\right.$ ) for the mode A. (a) Theoretical, (c) experimental uncontrolled. (b) Theoretical, (d) experimental controlled.

\section{Conclusions}

A piezoelectric patch shunted with a negative capacitance circuit has been used to simultaneously damp several modes of a square aluminum plate, reducing the sound radiation of the plate at low frequencies. Once the geometry is fixed the system has two degrees of freedom, dominated by the electrical parameters of the circuit: the resistance and the negative capacitance. We tune both the value of the negative capacitance, in order to place the structure close to the instability in the softener region, and the resistance of the circuit in such a way that the losses are enough to damp the several modes of the structure. Therefore, as the kinetic energy of the modes at these frequencies is reduced, 275

the power acoustic radiation is drastically attenuated. The problem is solved numerically and tested experimentally with good agreement. The results show the possibility of controlling the modal response of the system with a single shunted patch, opening prospects to improve the acoustic comfort. Moreover, the method shown in the article opens discussions about the direct optimization 
of the overall acoustic radiated power for the vibro-acoustic problems.

\section{Appendix A. Elastic and electric properties of the materials used in this work}

A detailed list of the elastic and electric parameters of the materials used in this work is shown as follows:

Table A.2: Elastic properties of the Aluminum.

\begin{tabular}{cccc}
\hline \hline Parameter & Symbol & Units & Value \\
\hline \hline Young Modulus & $E$ & GPa & 70 \\
Density & $\rho$ & kg.m ${ }^{-3}$ & 2700 \\
Poison's ratio & $\mu$ & & 0.33 \\
\hline \hline
\end{tabular}

Table A.3: Elastic properties of the PZ26.

\begin{tabular}{ccccc}
\hline \hline Parameter & Symbol & Units & Value \\
\hline \hline Density & $\rho$ & $\mathrm{kg} \cdot \mathrm{m}^{-3}$ & 7650 \\
Poison's ratio & $\mu$ & & 0.33 \\
\hline Elastic constants & $C_{11}^{E}$ & $\mathrm{~Pa}$ & 153.7 & $10^{9}$ \\
& $C_{12}^{E}$ & $\mathrm{~Pa}$ & 82.3 & $10^{9}$ \\
& $C_{13}^{E}$ & $\mathrm{~Pa}$ & 80.6 & $10^{9}$ \\
& $C_{33}^{E}$ & $\mathrm{~Pa}$ & 137.4 & $10^{9}$ \\
& $C_{44}^{E}$ & $\mathrm{~Pa}$ & 45.9 & $10^{9}$ \\
& $C_{66}^{E}$ & $\mathrm{~Pa}$ & 35.7 & $10^{9}$ \\
\hline \hline
\end{tabular}


Table A.4: Electric properties of the PZ26.

\begin{tabular}{cccc}
\hline \hline Parameter & Symbol & Units & Value \\
\hline \hline Dielectric constant & $\varepsilon_{11, r}^{S}$ & - & 1142 \\
& $\varepsilon_{33, r}^{S}$ & - & 668 \\
& $\varepsilon_{33, r}^{T}$ & - & 1097 \\
\hline Voltage coefficients & $e_{31}$ & C.m & -6.18 \\
& $e_{33}$ & C.m & 12.79 \\
& $e_{15}$ & C.m ${ }^{-2}$ & 12.88 \\
\hline \hline
\end{tabular}

\section{Appendix B. Negative Capacitance circuit: Electronic circuit with the operational amplifier}

In this work, the negative capacitance has been implemented experimentally by an analogical electronic circuit organized around an operational amplifier. Figure B.7 presents a picture of the circuit used in the experimental set-up and a schematic representation of the equivalent electrical circuit. The main interest of these circuits comes from the presence of potentiometers (blue components in Fig. B.7), that can be used to manually change the resistance values of the circuit which, combined with an operational amplifier have the ability to generate "negative" capacitance values. The equivalent resistance and the equivalent negative capacitance of the synthetic analogical circuit can be approximated by 34

$$
\begin{aligned}
R_{e q} & =R_{1}-\frac{R_{3} R_{2}}{R_{4}} \frac{\omega_{R C}^{2}}{\omega_{R C}^{2}+\omega^{2}} \equiv R^{e x p} \\
C_{e q} & =-\frac{R_{4}}{R_{3}} C_{2} \equiv C_{n e g}^{e x p}
\end{aligned}
$$

with $\omega_{R C}=1 / R_{2} C_{2}, R_{1}=40 \Omega, R_{2}=15 M \Omega, C_{2}=107 n F$ and $\omega$ the angular frequency. It is worth noting here that the ratio $\frac{\omega_{R C}^{2}}{\omega_{R C}^{2}+\omega^{2}}$ quickly tends toward 0 according to $\omega$ since $\omega_{R C}=6.23110^{-3}$. So $R^{\text {exp }} \simeq R_{1}$. In reality 
maximal resistance $R_{\text {pot,max }}=10 \mathrm{k} \Omega$ is used to control these two resistances. In fact, by modifying $R_{\text {pot }}$ we change these resistances as $R_{4}=R_{p o t}$ and $R_{3}=$ $R_{p o t, \max }-R_{\text {pot }}$. Therefore, the values of $R^{\exp }$ and $C_{\text {neg }}^{e x p}$ can be considered as constants in the target range of frequencies.

305 A controlled circuit allows us to detect the system instability by monitoring the saturation of the operational amplifier. The optimal value of the negative capacitance is obtained close to the instability.

This circuit allows us to set the negative capacitance close to the instability region by tuning the potentiometers. However, they present experimental constrains that limit the values of $C_{n e g}$ and $R$ close to the optimal values theoretically predicted. The experimental values used in this work are $C_{n e g}^{e x p} \simeq-45.610^{-9} F$ and $R^{\text {exp }}=39.9 \Omega$. They have been found from the values given form the numerical optimization procedure and then look around this values in order to find the good performance in the experimental set-up.

(a)

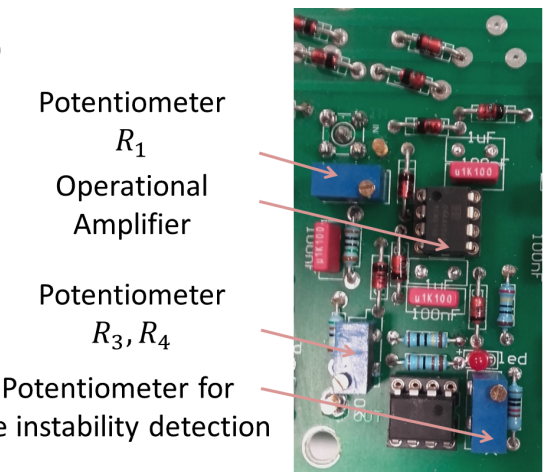

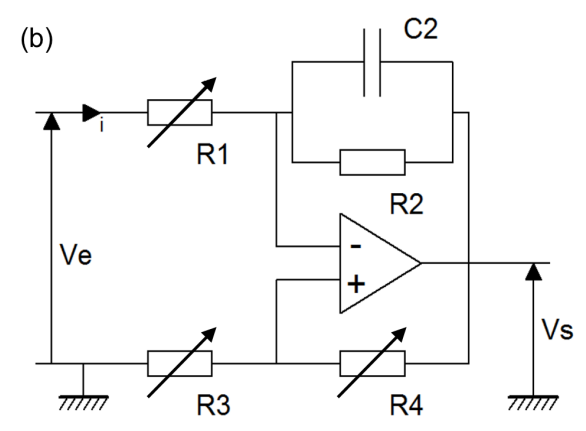

Figure B.7: (Color online) Picture (a) and schematic representation (b) of the analogical electrical circuit of the negative capacitance synthesis. 


\section{Appendix C. Control of modes B and C}

In addition to the results shown in the main manuscript we have evaluated the theoretical and the experimental maps of mobility for the modes at 1640 $\mathrm{Hz}$ (mode B) and $1950 \mathrm{~Hz}$ (mode C) for the controlled and uncontrolled cases. Figures C.8(a) and (b) show theoretical maps of mobility for the mode B in the uncontrolled and controlled cases respectively. Figures C.8(c) and (d) show theoretical maps of mobility for the mode $\mathrm{C}$ in the uncontrolled and controlled cases respectively. A significant reduction of the mobility can be seen for the controlled case.
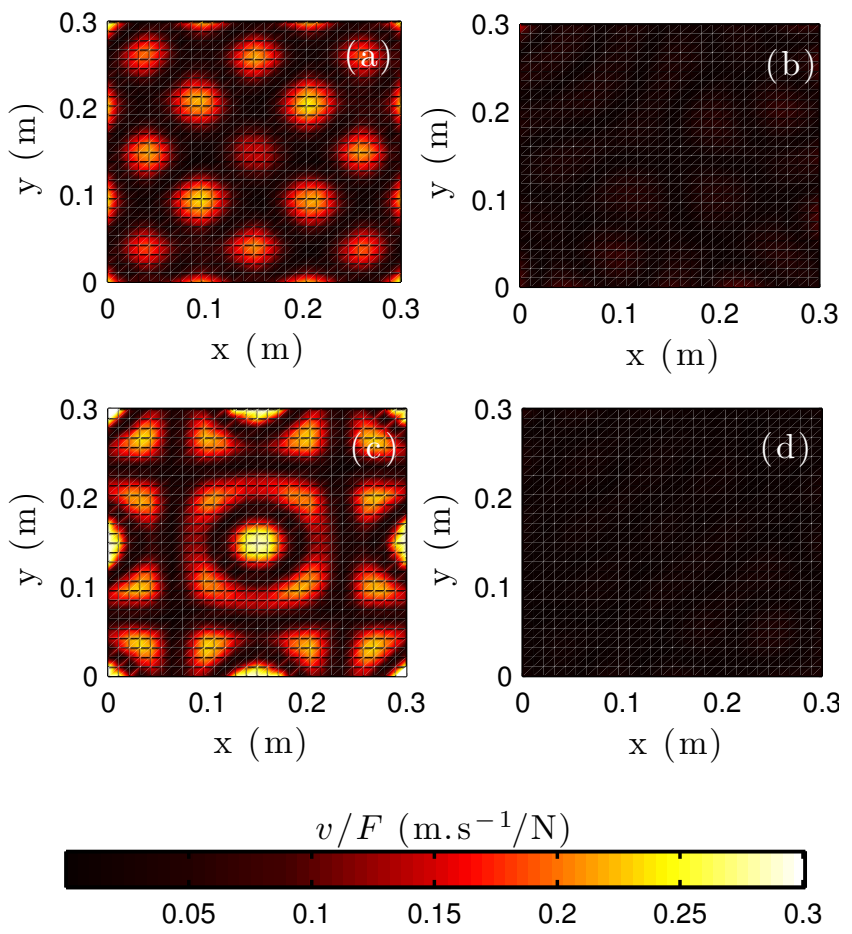

Figure C.8: (Color online) Velocity field normalized by the force excitation source $v / F$ for the modes B and C. (a) Theoretical uncontrolled for the mode B. (b) Theoretical controlled the mode B. (c) Theoretical uncontrolled for the mode C. (d) Theoretical controlled the mode C. 


\section{Acknowledgments}

This work is part of the Vibroleg project managed by IRT Jules Verne (French Institute in Research and Technology in Advanced Manufacturing Technologies for Composite, Metallic and Hybrid Structures). The authors wish to associate the industrial and academic partners of this project; respectively Airbus, STX, G.E., Daher, DCNS, Cetim, Bureau Veritas, LAUM and CNRS. V. Romero-García acknowledges the Metaplaque project founded by the RFI Le Mans Acoustique, project AICO/2016/060 by Consellera de Educacion, Investigacion, Cultura y Deporte de la Generalitat Valenciana and project FIS201565998-C2-2-P by Ministerio de Economía y Competitividad (Spain) and Euro-

\section{References}

[1] J. Hunt, J. Nissen, The broadband dynamic vibration absorber, J. Sound Vib. 83 (4) (1982) 573.

[2] G. Wang, X. Wen, J. Wen, L. Shao, Y. Liu, Two-dimensional locally resonant phononic crystals with binary structures, Phys. Rev. Lett. 93 (2004) 154302.

[3] T.-T. Wu, Z.-G. Huang, T.-C. Tsai, T.-C. Wu, Evidence of complete band gap and resonances in a plate with periodic stubbed surface, Appl. Phys. Lett. 93 (2008) 111902.

[4] M. Oudich, Y. Li, B. M. Assouar, Z. Hou, A sonic band gap based on the locally resonant phononic plates with stubs, New J. Phys. 12 (2010) 083049 .

[5] A. Khelif, B. Aoubiza, S. Mohammadi, A. Adibi, V. Laude, Complete band gaps in two-dimensional phononic crystal slabs, Phys. Rev. E 74 (2006) 
[6] S. Degraeve, C. Granger, B. Dubus, J.-O. Vasseur, M. P. Thi, A.-C. HladkyHennion, Bragg band gaps tunability in an homogeneous piezoelectric rod with periodic electrical boundary conditions, J. Appl. Phys. 115 (2014) 194508.

[7] N. Kherraz, L. Haumesser, F. Levassort, P. Benard, B. Morvan, Controlling Bragg gaps induced by electric boundary conditions in phononic piezoelectric plates, Appl. Phys. Lett. 108 (2016) 093503.

[8] R. Forward, Electronic damping of vibrations in optical structures, Appl. Opt. 18 (5) (1979) 690-697.

[9] N. Hagood, A. von Flotow, Damping of structural vibrations with piezoelectric materials and passive electrical networks, J. Sound Vib. 146 (1991) 243.

[10] O. Thomas, J. Ducarne, J. Deu, Performance of piezoelectric shunts for vibration reduction, Smart Mater. Struct. 21 (2012) 015008.

[11] S.-Y. Wu, Method for multiple mode piezoelectric shunting with single pzt transducer for vibration control, J. Intel. Mat. Syst. Str. 9 (1998) 991.

[12] S. Behrens, S. O. R. Moheimani, A. J. Fleming, Multiple mode current flowing passive piezoelectric shunt controller, J. Sound Vib. 266 (2003) 929.

[13] F. Casadei, B. S. Beck, K. A. Cunefare, M. Ruzzene, Vibration control of plates through hybrid configurations of periodic piezoelectric shunts, J. Intel. Mat. Syst. Str. 23 (10) (2012) 1169.

[14] S. Chen, G. Wang, J. Wen, X. Wen, Wave propagation and attenuation in plates with periodic arrays of shunted piezo-patches, J. Sound Vib. 232 (2013) 1520.

[15] L. Airoldi, M. Ruzzene, Design of tunable acoustic metamaterials through periodic arrays of resonant shunted piezos, New J. Phys. 13 (2011) 113010. 
[16] D. Casagrande, P. Gardonio, M. Zilletti, Smart panel with time-varying shunted piezoelectric patch absorbers for broadband vibration control, J. Sound Vib. 400 (2017) 288-304.

[17] B. Lossouarn, J.-F. Deü, M. Aucejo, K. A. Cunefare, Multimodal vibration damping of a plate by piezoelectric coupling to its analogous electrical network, Smart Mater. Struct. 25 (2016) 115042.

[18] J. Park, D. Palumbo, A new approach to identify optimal properties of shunting elements for maximum damping of structural vibration using piezoelectric patches, in: ACTIVE 04: International Symposium on Active Control of Sound and Vibration; Williamsburg, VA; United States, 2004., pp. 1-10.

[19] J. Becker, O. Fein, M. Maess, L. Gaul, Finite element-based analysis of shunted piezo- electric structures for vibration damping, Comput. Struct. 84 (31) (2006) 2340-2350.

[20] F. dell'Isola, C. Maurini, M. Porfiri, Passive damping of beam vibrations through distributed electric networks and piezoelectric transducers: prototype design and experimental validation, Smart Mater. Struct. 13 (2004) 299-308.

[21] C. Cross, S. Fleeter, Shunted piezoelectrics for passive control of turbomachine blading flow-induced vibrations, Smart Mater. Struct. 11 (2002) 239-248.

[22] M. Berardengo, S. Manzoni, A. M. Conti, Multi-mode passive piezoelctric shunt damping by means of matrix inequalities, J. Sound Vib. 405 (2017) 287-305.

[23] S. O. R Moheimani, A. J. Fleming, S. Behrens, Dynamics, stability, and control multivariable piezoelectric shunts, IEEE/ASME Transactions on Mechatronics, 9 (2004) 87-89. 
[32] B. de Marneffe, A. Preumont, Vibration damping with negative capacitance shunts: theory and experiment, Smart Mater. Struct. 17 (2008) 035015. 
[33] M. Berardengo, O. Thomas, C. Giraud-Audine, S. Manzoni, Improved resistive shunt by means of negative capacitance: new circuit, performances and multi-mode control, Smart Mater. Struct. 25 (2016) 075033.

[40] K. Mulholland, The effect of sound-absorbing materials on the sound insulation of single panels., Appl. Acoust. 2 (1) (1969) 1-7.

[41] S. Brown, J. Niedzielski, G. Spalding, Effect of sound-absorptive facings 455 on partition airborne-sound transmission loss., J. Acoust. Soc. Am. 63 (6) (1978) 185101856.

[42] D. Takahashi, Sound transmission through single plates with absorptive facings., J. Acoust. Soc. Am. 83 (4) (1988) 1453-1457. 
[43] M. Ahmadian, K. Jeric, On the application of shunted piezoceramics for increasing acoustic transmission loss in structures., J. Sound Vib. 243 (2) (2001) 347-59.

[44] B. Petitjean, I. Legrain, F. Simon, S. Pauzin, Active control experiments for acoustic radiation reduction of a sandwich panel: feedback and feedforward investigations, J. Sound Vib. 252 (1) (2002) 19-36.

[45] M. Ozer, T. Royston, Passively minimizing structural sound radiation using shunted piezo-electric materials., J. Acoust. Soc. Am. 114 (4) (2003) 193446.

[46] S. Pietrzko, Q. Mao, Reduction of structural sound radiation and vibration using shunt piezoelectric materials., Solid State Phenom. 2009;147-149:8829. 147-149 (2009) 882-889.

[47] O. Thomas, J. Deü, J. Ducarne, Vibrations of an elastic structure with shunted piezoelectric patches: efficient finite elements formulation and electromechanical couplings coefficients., Int. J. Numer. Meth. Eng. 80 (2) (2009) 235-68.

[48] G. Rosi, J. Pouget, F. dell'Isola, Control of sound radiation and transmission by a piezoelectric plate with an optimized resistive electrode., Eur. J. Mech. A-Solid 29 (2010) 859-70.

[49] W. Larbi, L. P. da Silva, J.-F. Deü, An efficient fe approach for attenuation of acoustic radiation of thin structures by using passive shunted piezoelectric systems, Appl. Acous. 128 (2017) 3-13.

[50] C. E. Wallace, Radiation resistance of a rectangular panel, J. Acoust. Soc. Am. 51 (1972) 946. 